\title{
AVALIAÇÃO DOS NÍVEIS DE NITRATO E NITRITO EM SALSICHAS COMERCIALIZADAS NA CIDADE DE LAVRAS - MG
}

\author{
Hebe Mariane Freire FERREIRA ${ }^{1}$ \\ Edimar Agnaldo MOREIRA ${ }^{2}$ \\ Daniela Fernanda FREITAS ${ }^{3,4}$
}

\begin{abstract}
${ }^{1}$ Discente, Centro Universitário de Lavras, Curso de Graduação em Farmácia Generalista; hebe.freire@ yahoo.com.br; ${ }^{2}$ Discente, Universidade Federal de Alfenas, Pós Graduação em Ecologia e Tecnologia Ambiental; edi.scarab@gmail.com

${ }^{3}$ Docente, Centro Universitário de Lavras, Curso de Graduação em Farmácia Generalista; danielaffreitas @bol.com.br ${ }^{4}$ Docente, Faculdade de Ciências e Tecnologias de Campos gerais, Curso de Graduação em Farmácia Generalista.
\end{abstract}

\section{Recebido em: 21/10/2013 - Aprovado em: 20/12/2013 - Disponibilizado em: 15/01/2014}

RESUMO: O nitrito possui um impacto nocivo sobre a saúde humana, devido à sua reação com aminas secundárias. Deste modo, é essencial a determinação de nitrato e nitrito em amostras de alimentos, como embutidos de carne, que são amplamente consumidos pela população em geral, principalmente pelas crianças. Assim, o objetivo deste trabalho foi o de verificar a presença dessas substâncias em salsichas comercializadas em Lavras-MG por método descrito pela AOAC (Official Methods of Analysis of the Association of Official Analytical Chemists). Foram coletados três tipos de amostras de salsichas (A, B e C). As amostras foram analizadas no espectrofotômetro ( $\lambda 474 \mathrm{~nm})$. Para o cálculo do teor de nitrato e nitrito utilizou-se a equação $(Y=0,1734 X+0,0097)$, com um coeficiente de determinação $(\mathrm{R} 2)$ de 0,9999 . Foram encontrados valores entre $1,6725-2,6011 \mathrm{mg} / \mathrm{Kg}$ e $1,5876-3,1332 \mathrm{mg} / \mathrm{Kg}$ de nitrato e nitrito respectivamente na amostra A; 0,0922 - 0,2044 mg/Kg e 0,6845 - 1,5761 mg/Kg na amostra B e 0,0638 - 3,2842 mg/Kg e 1,1839 $1,6683 \mathrm{mg} / \mathrm{Kg}$ na amostra C. Este estudo sugerem que, as amostras estão em concordância com as exigências legais quando se compara com o limite máximo de resíduo permitido para salsicha $0-3,7 \mathrm{mg} / \mathrm{Kg}$ de nitrato, porém para os valores de nitrito percebe-se que os dados obtidos ultrapassaram o limite permitido pela legislação, que é de 0 - 0,07 $\mathrm{mg} / \mathrm{Kg}$ de nitrito.

PALAVRAS CHAVE. Nitrato. Nitrito. Alimentos.

\begin{abstract}
Nitrite has a harmful impact on human health due to its reaction with secondary amines, which are naturally present in foods, forming N-nitrosamines, compounds with carcinogenic potential. Thus, it is essential the determination of nitrate and nitrite in food samples, such as built-in meat products which are widely consumed by the general population, especially by children. The objective this work was to verify the presence of these substances in sausages sold in Lavras-MG by the method described by AOAC (Official Methods of Analysis of the Association of Official Analytical Chemists). Were collected three types of sausage samples (A, B and C). The samples were analyzed in a spectrophotometer $(\lambda 474 \mathrm{~nm})$. For the calculation of the nitrate and nitrite levels it was used the equation $(\mathrm{Y}=$ $0.1734+0.0097 \mathrm{X}$ ) with a coefficient of determination (R2) 0.9999. It was found values between 1.6725 to $2.6011 \mathrm{mg} /$ $\mathrm{kg}$ and 1.5876 to $3.1332 \mathrm{mg} / \mathrm{kg}$ of nitrate and nitrite respectively at sample A; 0.0922 to $0.2044 \mathrm{mg} / \mathrm{kg}$ and 0.6845 to 1 , $5761 \mathrm{mg} / \mathrm{kg}$ in sample B and 0.0638 to $3.2842 \mathrm{mg} / \mathrm{kg}$ and 1.1839 to $1.6683 \mathrm{mg} / \mathrm{kg}$ in the sample C. This study suggest that the samples are consistent with legal requirements when compared with the maximum residue limit allowed for sausage from 0 to $3.7 \mathrm{mg} / \mathrm{kg}$ of nitrate, but for nitrite values it was realized that data obtained exceeded the limit allowed by law, which is $0-0.07 \mathrm{mg} / \mathrm{kg}$ nitrite.
\end{abstract}

KEYWORDS. Nitrate. Nitrite. food.

\section{INTRODUÇÃO}

Os alimentos são essenciais à saúde do

indivíduo, porém o consumo de alimentos

contendo agentes químicos, que não sejam nutrientes, pode levar ao aparecimento de

diversos efeitos classificados como: efeitos

tóxico, nocivo, danoso, adverso ou não

esperado (MIDIO; MARTINS, 2000). 
Estes efeitos, não podem ser classificados, uma vez que adquirem características próprias sujeita ao organismo atingido. Os efeitos são, tipicamente, doses dependentes (concentração - dependentes), ou seja, quanto maior a concentração do agente químico no alimento maior a magnitude ou possibilidade de ocorrência do efeito tóxico ou reação tóxica. Os efeitos tóxicos produzidos por substâncias não-nutricionais, presentes em alimentos se classificam quanto à gravidade dos sinais e sintomas como: leves, moderados ou severos, e quanto à velocidade em que ocorrem se classificam como: agudo, subagudo, subcrônico ou crônico (MIDIO; MARTINS, 2000).

Acredita-se que a maior fonte de contaminação de alimentos por agentes químicos seja aquela oriunda da necessidade de aumento da atividade agropecuária, como a aplicação de fertilizantes, praguicidas, promotores do crescimento animal e vegetal e quimioterápico de importância veterinária (MIDIO; MARTINS, 2000).

A análise de resíduos inclui a segurança alimentar e esta determina se o alimento é seguro ou não ao consumo humano. Testes são denominados para garantir que os resíduos estejam a níveis que respeitem os limites máximos permitidos e de acordo com as legislações (BRASIL, 1999;

O'KEEFE, 2000).

A toxicologia de alimentos estuda os efeitos nocivos provocados por substâncias químicas visando definir os limites e condições de exposição seguras na ingestão de alimentos que apresentam certo grau de contaminação. Para a avaliação da toxicidade de um agente tóxico presente em um alimento devem-se observar as condições de exposição, como a concentração do agente tóxico presente, a frequência o tempo em que é ingerida, a via de introdução no organismo e a suscetibilidade do organismo (MIDIO; MARTINS, 2000).

A exposição a essas substâncias tóxicas através do consumo de alimentos envolve riscos a saúde do consumidor, este fato justifica a necessidade de divulgar informações à população sobre os níveis de resíduos instituírem os riscos que essas substâncias oferecem. A partir destas 
informações, medidas de vigilância sanitária, poderão ser definidas e adotadas pelas agências governamentais que lidam com a saúde pública.

Entre as diversas substâncias químicas capazes de provocar problemas pela ingestão de alimentos estão os nitratos e nitritos. Sais de nitrato e nitrito estão naturalmente presentes no ambiente, na água e em alimentos de origem vegetal, ou são adicionados intencionalmente durante o processamento de alimentos de origem animal (ARAÚJO; MIDIO, 1990; DUARTE; MIDIO, 1996).

A utilização destes sais tem por finalidade conferir cor e sabor aos produtos embutidos, além de funcionar como agente bacteriológico. Alguns artigos relatam que o nitrito de sódio possui capacidade de inibir o crescimento e a produção de toxinas da espécie de Clostridium, porém há indícios que o calor é mais eficiente ao combate destes microrganismos que o próprio nitrito. A importância do nitrato para a saúde humana está relacionada devido ao fato do nitrato ser facilmente convertido a nitrito. Os efeitos tóxicos mais importantes decorrentes da ingestão de nitrato e de nitrito são a metemoglobinemia, em neonatos e em indivíduos com deficiência congênita de metemoglobina-redutase ou deficiência de glicose-6-fosfato desidrogenase (G6PD). Também pode ocorrer a formação in vivo de $\mathrm{N}$-nitrosaminas, que podem estar associadas com um alto risco de câncer gástrico, hepático e de esôfago (GANGOLLI et al., 1994).

Dado esta importância, o objetivo do presente trabalho foi de verificar qualitativamente a presença de nitrato e nitrito em amostras de salsichas comercializadas na região de Lavras/MG. Os valores obtidos foram comparados com os valores permitidos pela Legislação Brasileira.

\section{MATERIAL E MÉTODOS}

$$
\text { A pesquisa fundamentou-se em }
$$
verificar a presença de nitrato e nitrito em salsichas comercializadas na cidade de Lavras/MG. Todos os testes foram realizados no campus do UNILAVRAS com o apoio financeiro da Fundação de Amparo à Pesquisa do Estado de Minas Gerais (FAPEMIG). Os 
setores onde foram realizados os testes foram

o Laboratório de Pesquisa I e Laboratório de Química e Ciências Farmacêuticas.

Para o presente estudo, foram adquiridas três marcas de salsichas comercializadas a granel no comércio de Lavras/MG. Após a coleta, as amostras foram submetidas à refrigeração a $4 \mathrm{oC}$ até $\mathrm{o}$ momento da análise.

O método utilizado foi descrito pela AOAC (Official Methods of Analysis of the Association of Official Analytical Chemists), que fundamenta-se na redução convencional do nitrato a nitrito em coluna de cádmio, com posterior análise espectrofotométrica. Conforme Dennis et al. (1990), foram realizadas as etapas de desproteinização das amostras utilizando uma solução de bórax e a de clarificação feita através do ferrocianeto de potássio e acetato de zinco. O nitrito foi determinado espectrofotometricamente a 474 nm após diazotação. Para a reação de diazoconjugação, na formação do cromógeno, pode ser utilizado o reativo de ácido sulfanílico ou alfa naftol com solução tampão de pH 9,6 - 9,7 conforme as normas analíticas do Instituto Adolfo Lutz (1985).

Assim, o nitrato foi determinado após redução quantitativa a nitrito, utilizando-se grânulos de cádmio revestidos com cobre (CORTAS; WAKID, 1990).

Foram montadas colunas de cádmio utilizando barras de zinco (cerca de seis barras) estas foram colocadas em um béquer de 1000 mL, e imersas em uma solução 2,7\% de sulfato de cádmio. Depois de aproximadamente 20 horas, é observado completa a reação de troca entre o zinco e o cádmio da solução. Esta é cessada a partir do momento em que a solução se torna saturada. $\mathrm{Zn}+\mathrm{CdSO} 4 \rightarrow \mathrm{ZnSO} 4+\mathrm{Cd}:$ Reação de formação de cádmio. Fonte: SWANN, 1995 apud ANDRADE, 2004.

Desta maneira o cádmio, depositado sobre as barras, foi raspado e coletado com uma pequena porção da solução do béquer, para uma melhor conservação. $\mathrm{O}$ cádmio em grânulos foi triturado no liquidificador por 3 segundos, logo após foi transferido para uma coluna que é composta por uma bureta de 50 $\mathrm{mL}$, grumos de cádmio e um disco em 
micropore para sustentar os grumos de cádmio dentro da bureta. A coluna foi preenchida com o cádmio, e este lavado com 100 mL de $\mathrm{HCl}$ 0,1 mol L-1, solução esta utilizada para conservação do cádmio. Importante que o armazenamento da coluna seja sob abrigo da luz para evitar processos de oxidação.

A técnica de desproteinização foi utilizada para tratar as amostras antes da passagem pela coluna. Esta técnica consistiu em pesar cuidadosamente $10 \mathrm{~g}$ de salsicha e adicionar a este $40 \mathrm{~mL}$ de água e $5 \mathrm{~mL}$ de solução I, aquecer à $800 \mathrm{C}$, agitando ocasionalmente por 15 minutos a solução. Resfriar a amostra até que chegue à temperatura ambiente, adicionando $2 \mathrm{~mL}$ da solução II e 2 mL da solução III, agitando vigorosamente após cada adição durante 5 minutos. Transferir a amostra para um balão de fundo chato de $100 \mathrm{~mL}$ e deixar em repouso durante 30 minutos.

Após repouso, o volume foi completado para $100 \mathrm{~mL}$ com água deionizada, agitando vigorosamente e filtrando em papel livre de nitrito e nitrato, para então se determinar a concentração de nitrito presente nesta solução.

Logo após, o processo de ativação da coluna foi iniciado a análise com a passagem de 40 mL de solução básica concentrada, $40 \mathrm{~mL}$ de solução padrão estoque de nitrato e $40 \mathrm{~mL}$ de solução básica diluída. Após esse processo, a coluna foi carregada com $20 \mathrm{~mL}$ da solução desproteinizada (amostra) com $5 \mathrm{~mL}$ da solução tampão. A velocidade de passagem foi de $5 \mathrm{~mL} / \mathrm{min}$, desprezando os primeiros 10 $\mathrm{mL}$ da solução. $\mathrm{O}$ filtrado restante foi recolhido em um balão volumétrico de 100 $\mathrm{mL}$, completando o volume com água deionizada, que também passa pela coluna.

Após a passagem da amostra, a coluna de cádmio foi regenerada, com as seguintes soluções: $20 \mathrm{~mL}$ de solução básica concentrada, $20 \mathrm{~mL}$ da solução padrão estoque de nitrato $100 \mathrm{mg} \mathrm{L}-1,30 \mathrm{~mL}$ de água, $30 \mathrm{~mL}$ de solução básica concentrada, 20 mL de água e por último 20 mL de solução básica diluída. Para reiniciar o processo, logo após a sua regeneração, foram eluídos $25 \mathrm{~mL}$

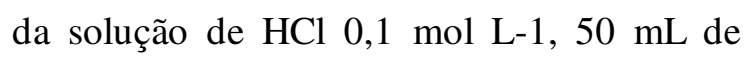
água e $25 \mathrm{~mL}$ de uma solução de tampão 
diluído 1:9. Para determinação da presença de nitrato e nitrito em salsichas, foi preciso preparar uma solução desproteinizada e posteriormente obter o eluato, para que se fosse possível fazer a leitura em espectrofotômetro.

O eluato só foi obtido a partir da solução desproteinizada que foi passada pela coluna de cádmio para redução do nitrato presente na amostra em nitrito e o nitrato que fortificou a amostra se reduza a nitrito. Somente o nitrito é capaz de reagir com a solução de alfa naftol (reagente cromogênico), é esta a razão de se montar novas colunas de cádmio para obtenção de nitrito a partir do nitrato que esteja presente na amostra.

O alfa naftol na presença de nitrito torna a solução alaranjada, quando isto ocorre é fato que a amostra tenha nitrito presente.

\section{Determinação de nitrito:}

Aos 10 mL da solução desproteinizada foram adicionados $5 \mathrm{~mL}$ de tampão e $10 \mathrm{~mL}$ de alfanaftol. Deixando em estufa por 30 minutos a $30^{\circ} \mathrm{C}$ e esfriado a temperatura ambiente. Após esfriar, foi realizada a leitura a $474 \mathrm{~nm} \mathrm{e}$ calculado o valor de nitrito presente na amostra, usando a curva analítica previamente estabelecida.

\section{Determinação de nitrato:}

Aos $10 \mathrm{~mL}$ do eluato da coluna de cádmio foram adicionados $5 \mathrm{~mL}$ de tampão e $10 \mathrm{~mL}$ de alfa-naftol. Deixando em estufa por $30 \mathrm{~min}$ a $30^{\circ} \mathrm{C}$ e, após esse tempo foi esfriada à temperatura ambiente e realizada a leitura a $474 \mathrm{~nm}$, seguido do cálculo do valor de nitrato através da equação:

$\mathrm{O}$ nitrato presente na amostra foi calculado a partir da equação:

Nitrato amostra $=(A-B) \times 1,231$

Onde:

$\mathrm{A}=$ somatória de nitrato e nitrito, lido no eluato da coluna, sob a forma de nitrito;

$\mathrm{B}=$ teor de nitrito, lido diretamente no desproteinizado, sem passar pela coluna; 1,231= fator de correção para a diferença de massa molecular entre nitrito e nitrato.

Para avaliação dos resultados obtidos pela pesquisa realizada, foi necessário 
preparar uma Curva Analítica, onde diluiu-se a solução de nitrato padrão em água deionizada.

\section{RESULTADOS E DISCUSSÃO}

\section{A Figura 1 ilustra a Curva Analítica} utilizada para calcular os resultados da pesquisa. Cada ponto corresponde à média dos valores obtidos na análise de cinco replicatas, sendo cada replicata com $0,2 \mathrm{~mL}$, $0,4 \mathrm{~mL}, 0,8 \mathrm{~mL}$ e $1,6 \mathrm{~mL}$ de solução padrão de nitrato. Os dados apresentados evidenciam linearidade satisfatória no intervalo selecionado, com coeficiente de determinação de 0,9999 .

Figura 1 - Curva Analítica para alíquotas de Solução de nitrito de sódio padrão. Fonte - Ferreira (2011).

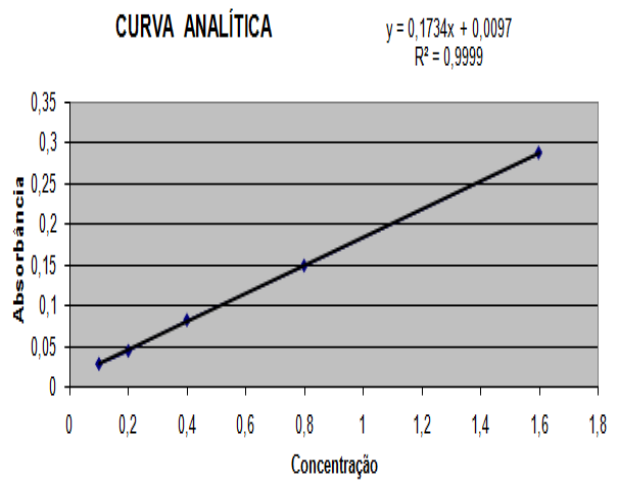

O Quadro 1 mostra os resultados obtidos a partir da solução desproteinizada e eluato obtido após a passagem das amostras pela coluna de cádmio.

O nitrato é uma substância química que dá origem ao nitrito, seu metabólito. O nitrato é uma fonte preocupação para o mercado consumidor, mas não tão preocupante quanto à presença do nitrito como conservante em alimentos.

Quadro 1: Teor de nitrito e nitrato nas amostras analisadas. Fonte - Ferreira (2011).

\begin{tabular}{|c|c|c|}
\hline AMOSTRAS & $\begin{array}{c}\text { NITRITO } \\
(\mathbf{m g} / \mathbf{K g})\end{array}$ & $\begin{array}{c}\text { NITRATO } \\
(\mathbf{m g} / \mathbf{K g})\end{array}$ \\
\hline A 1 & 3,1332 & 1,6725 \\
\hline A 2 & 3,0813 & 1,9367 \\
\hline A 3 & 1,5876 & 2,6011 \\
\hline A 4 & 1,6568 & 2,5159 \\
\hline B 1 & 0,6845 & 0,1345 \\
\hline B 2 & 1,2993 & 0,0922 \\
\hline B 3 & 1,1263 & 0,1206 \\
\hline B 4 & 1,5761 & 0,2044 \\
\hline C 1 & 1,1839 & 0,1816 \\
\hline C 2 & 1,262 & 3,2842 \\
\hline C 3 & 1,6528 & 0,0829 \\
\hline C4 & 1,6683 & 0,0638 \\
\hline
\end{tabular}

O Gráfico 1 compara os valores obtidos de nitrato em 3 marcas $\mathrm{A}, \mathrm{B}$ e $\mathrm{C}$, sendo cada uma composta por 4 lotes diferentes.

O valor de nitrato nas amostras recomendado é de $0-3,7 \mathrm{mg} / \mathrm{kg}$ e observa-se que das amostras analisadas, todas possuem 
nitrato dentro do valor de referência para o consumo humano. O limite recomendado para a presença de nitrato em embutidos é um valor maior quando comparado ao valor especificado para nitrito, isto pode ser justificado pelo fato de que o nitrato não é tão prejudicial ao organismo quanto o nitrito, que em pequenas quantidades consegue ser eficiente para provocar efeitos tóxicos.

Gráfico 1: Comparação do valor obtido de nitrato com o valor de referência. Fonte - Ferreira (2011).

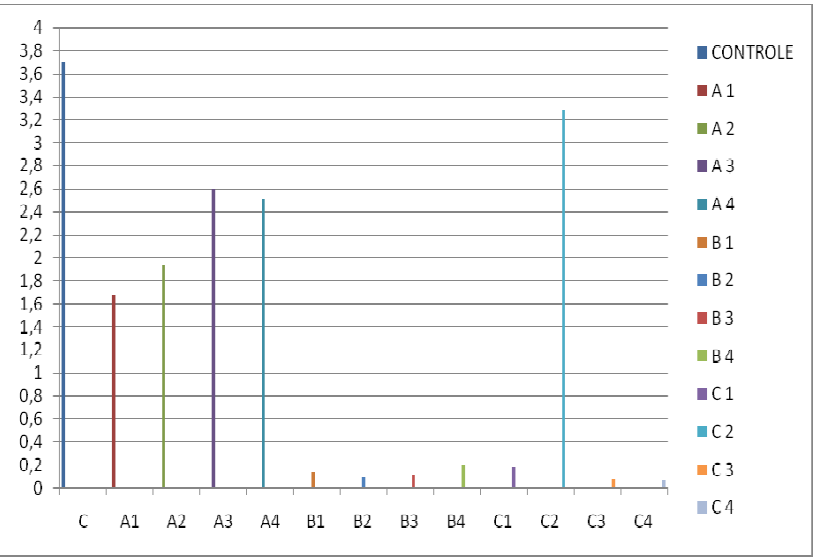

Em um estudo análogo ao presente trabalho, Melo Filho et al. (2004), avaliou a qualidade de salsichas comercializadas em Recife-PE. Os níveis de nitrito residual e nitrato foram determinados em 54 amostras de salsichas, coletadas segundo região de origem e marca, de acordo com 3 grupos e 9 sub-grupos. Foram detectados níveis de nitrato acima do que é permitido (grupo (C) $67 \%$ das amostras apresentavam-se com nitrito residual $>150 \mathrm{mg} / \mathrm{kg}$ ( $18 \%$ do total); e os níveis de nitrato $>300 \mathrm{mg} / \mathrm{kg}$ em $17(\mathrm{~A})$, 67(B) e $83 \%$ (C) das amostras $(\mathrm{p}<0,05)$, acima do nível máximo permitido). Os resultados permitiram indicar que os níveis de nitrato e nitrito em salsichas representam um risco potencial à saúde do consumidor devido ao não cumprimento da legislação na produção e comercialização desse produto.

O Gráfico 2 compara os valores obtidos de nitrito nas marcas A, B e C, sendo cada uma composta por 4 lotes diferentes. Gráfico 2: Comparação do valor obtido de nitrito com o valor controle. Fonte - Ferreira (2011).

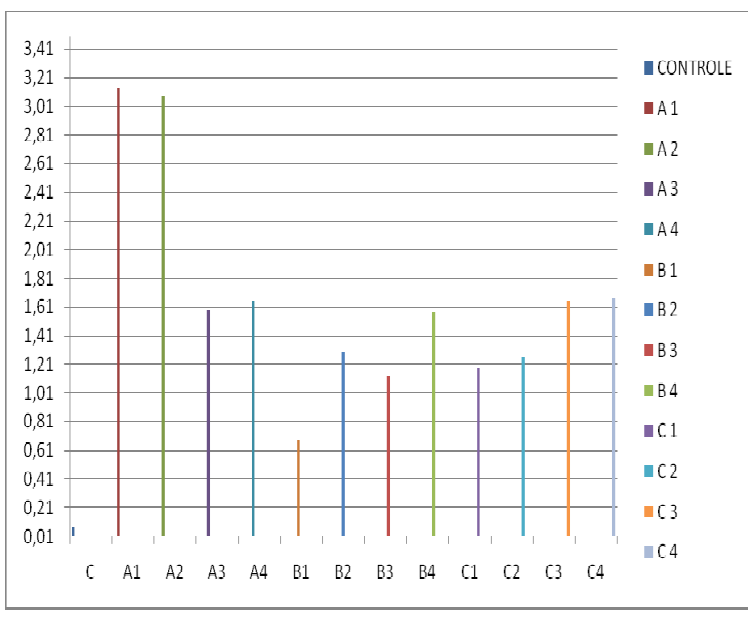

O valor de nitrito nas amostras recomendado é de $0-0,07 \mathrm{mg} / \mathrm{kg}$ e observase que das amostras analisadas, todas 
ultrapassaram o limite de nitrito que é permitido.

Este fato pode ser justificado pela grande facilidade em que o nitrato é convertido a nitrito. Comparando-se os valores de referência de nitrato/nitrito, observa-se que o valor de referência para nitrato é maior e isto influencia na quantidade final obtida de nitrito. O preocupante neste fato é que o valor de referência para a presença de nitrito é muito baixo e se o nitrato for eficientemente reduzido a nitrito, os valores obtidos de nitrito não estarão dentro do valor de referência e isto aumenta a possibilidade de aparecimento de efeitos tóxicos.

Os efeitos tóxicos produzidos por estes conservantes são consequências da presença de nitrito no organismo, já que a concentração de nitrito, quando alta, é influenciadora na formação de $\mathrm{MeHb}$, que posteriormente poderá provocar cianose até efeitos fatais. A presença de nitrito, também influencia a formação de compostos N-nitrosos, fatores estimulante para o surgimento do câncer, principalmente câncer gástrico, hepático e de esôfago.
Ainda foram realizados testes, para verificar a eficiência da coluna de cádmio quanto à redução de nitrato a nitrito, já que o nitrito é a substância capaz de ser identificada pelo alfanaftol, responsável pela coloração da solução.

\section{CONCLUSÃO}

De acordo com os dados deste trabalho amostras encontram-se dentro dos valores estabelecidos pela IDA no Brasil e Mercosul para nitratos, porém para nitritos, os valores ultrapassam os valores de referência estabelecidos e recomendados pela FAO/OMS.

A análise periódica dos teores de nitratos e nitritos constituiu-se ferramenta importante para o controle de qualidade dos alimentos ingeridos pela população, pois podem causar efeito tóxico em indivíduos expostos através de alimentos, dependendo da quantidade ingerida e da susceptibilidade do organismo.

\section{REFERÊNCIAS BIBLIOGRÁFICAS}

ARAUJO, A.C.P.; MÍDIO, A.F. Nitratos y nitritos em alimientos infantiles industrializados y caseros. Alimentaria, 1990. v.27, n.209, p.69-75.

BRASIL. Ministério da Agricultura, Pecuária e Abastecimento. Instrução Normativa 
número 42 de 20 de dezembro de 1999, disponível em:<

http://extranet.agricultura.gov.br/sislegisconsu lta/consultarLegislacao.do?operacao=visualiz ar\&id=16717 >Acesso em 15/11/2012.

CORTAS, N. K.; WAKID, N. W., Determination of inorganic nitrate in serum and urine by a kinetic cadmium reduction method. Clinical Chemistry, 1990. v.36, n.8, p.1440-1443.

DUARTE, M.; MÍDIO, A.F. Nitratos e nitritos em Alimentos. Cadernos de Nutrição, 1996. v.12, p.19-30.

GANGOLLI, S.D.; VAN DEN BRANDT, P.A.; FERON, V.J.; JANZOWSKY, C.; KOEMAN, JH et al. Nitrate, nitrite and Nnitroso coumponds. European Journal of Pharmacology, 1994. 292 p..

MELO FILHO, A. B. de; BISCONTINI, T.M. B.; ANDRADE, S. A. C. Níveis de nitrito e nitrato em salsichas comercializadas na região metropolitana do Recife. Ciência e tecnologia de alimentos. Campinas, 2004. v.24, n. 3, p. 390-392.

MIDIO,A.F.; MARTINS,D.I. Toxicologia de Alimentos. São Paulo: Livraria Varela, 2000. $295 p$.

O'KEEFE, M. Residue Analysis in Food. Principles and Applications, Harwood Academic Publishers, Amsterdam, The Netherlands, 2000. 